\title{
ЗАВИСИМОСТЬ ХАРАКТЕРИСТИК ДЕТОНАЦИОННЫХ ВОЛН В МНОГОКОМПОНЕНТНОЙ ПУЗЫРЬКОВОЙ ЖИДКОСТИ ОТ НАЧАЛЬНОГО ДАВЛЕНИЯ
}

\author{
Гималтдинов Ильяс Кадирович', \\ iljas_g@mail.ru \\ Левина Татьяна Михайловна', \\ tattin76@mail.ru \\ Кучер Анастасия Михайловна², \\ kucheram85@mail.ru \\ 1 Уфимский государственный нефтяной технический университет, \\ Россия, 450062, Республика Башкортостан, г. Уфа, ул. Космонавтов, 1. \\ 2 Башкирский государственный университет, \\ Россия, 453100, г. Стерлитамак, пр. Ленина, 49.
}

\begin{abstract}
Актуальность. Пузырьковые жидкости являются распространенной рабочей средой в ряде отраслей народного хозяйства. Одним из интереснейших процессов, происходящих в пузырьковой жидкости с взрывчатым газом внутри пузырьков, является пузырьковая детонация - распространение детонационных волн. Детонационные волны также могут распространяться в жидкости с пузырьками, частично состоящими из инертного газа. В этом случае наличие пузырьков с инертным газом является некоторым управляющим параметром для характеристик (амплитуды, протяженности, пределов распространения) детонационной волны. Кроме того, существуют экспериментальные данные о влиянии первоначального давления на характеристики волн пузырьковой детонации в многокомпонентной пузырьковой жидкости. Это обусловливает необходимость исследования детонационных волн в многокомпонентных пузырьковых системах при различных значениях начального давления.

Цель исследования: изучить динамику детонационных волн в пузырьковой жидкости, частично состоящую из пузырьков с неактивным (не горючим) газом при различных значениях начального давления.

объект: детонационные волны в пузырьковой жидкости, содержащей пузырьки с взрывчатым (активным) и негорючим (неактивным) газом.

Методика исследования базируется на фундаментальных уравнениях механики многофазных сред, которые решаются численным методом.

Результаты исследования по выявлению особенностей распространения детонационных волн в многокомпонентной пузырьковой жидкости при различных начальных давлениях позволили сделать выводы и дать рекомендации. С уменьшением начального давления многокомпонентных пузырьковых сред скорость распространения детонационных волн снижается. Зависимость скорости детонационной волны в многокомпонентной пузырьковой жидкости от начального давления близка к линейной.
\end{abstract}

\section{Ключевые слова:}

Нефтегазовое производство, взрывобезопасность, детонационные волны, пузырьковая жидкость, начальное давление.

\section{Введение}

Детонационные процессы существуют в различных средах. Несмотря на различие физико-химических свойств систем, процесс детонации обладает общим признаком: детонация - самоподдерживающийся процесс [1-3]. Это связано с тем, что детонационный процесс протекает в средах, когда в них может возникать взрывное выделение энергии. Возможность существования волн детонации обеспечивается энерговыделением в среде.

Распространение детонационных волн в пузырьковых жидкостях - уникальное явление, т. к. в этом случае детонация инициируется и распространяется в среде с очень низкой калорийностью. При этом распространение детонационных волн в жидкостях с пузырьками, обладая схожими для всех взрывных процессов признаками, имеет ряд особенностей, проявляющихся в свойствах и структуре волн [4-11].

Получение высоких температур в газе, помещенном в жидкости в виде одного пузырька или пузырькового кластера, представляет как теорети- ческий, так и практический интерес [12-14]. Исследованию динамики детонационной волны в многокомпонентной пузырьковой жидкости посвящена работа [15], где изучены особенности сжатия пузырьков с инертным газом детонационными волнами. Показано, что детонационные волны являются средством сжатия инертных пузырьков во всей области расчета, в то время как граничное давление может сжать пузырьки только около участков вблизи границы, через которую вносится возмущение.

Кроме этого, присутствие в жидкости наряду с пузырьками химически активного газа пузырьков химически неактивного газа уменьшает энергосодержание среды. Экспериментальному изучению детонационных волн в пузырьковых средах, частично состоящих из инертного газа, посвящена работа [16]. В этой работе исследованы структура, свойства и критические условия возбуждения волн пузырьковой детонации в таких средах. Кроме того, установлены основные закономерности действия волны пузырьковой детонации на пузырьки 
с активным и инертным газом. Экспериментальному исследованию влияния изменения начального давления пузырьковых жидкостей на свойства детонационной волны в пузырьковой жидкости посвящены работы $[17,18]$. В работе [19] теоретически изучено влияние первоначального давления в пузырьковой системе на характеристики (скорость, амплитуду и т. д.) детонационных волн.

В данной работе исследуется влияние первоначального давления в многокомпонентной пузырьковой системе, состоящей из пузырьков активного (горючего) и неактивного (негорючего) газа, на характеристики детонационных волн.

\section{Постановка задачи и основные уравнения}

Исследуем распространение волны в пузырьковой жидкости. Положим, что пузырьковая жидкость состоит из двух сортов пузырьков: активных (заполненных горючим газом, например ацетилено-кислородной смесью) и пассивных (заполненных негорючим газом, например воздухом). В пузырьках, содержащих взрывчатый газ, при достижении температуры воспламенения газа $T$ * происходит реакция, сопровождающаяся выделением тепла и увеличением температуры на величину $\Delta T$, которая соответствует калорийности газовой смеси. С учетом допущений, принятых выше, система уравнений, состоящая из уравнений сохранения масс, числа пузырьков, импульсов и давления газа в обоих типах пузырьках $[14,15,20]$, имеет вид:

$$
\begin{gathered}
\frac{d \rho_{i}}{d t}+\rho_{i} \frac{\partial v}{\partial x}=0(i=l, g), \frac{d n_{j}}{d t}+n_{j} \frac{\partial v}{\partial x}=0(j=1,2), \\
\rho_{l}^{0} \frac{d v}{d t}+\frac{\partial p_{l}}{\partial x}=0, \\
\frac{d p_{g j}}{d t}=-\frac{3 \gamma_{j} p_{g j}}{a_{j}} w_{j}-\frac{3\left(\gamma_{j}-1\right)}{a_{j}} q_{j}, \\
\frac{d}{d t}=\frac{\partial}{\partial t}+v \frac{\partial}{\partial x}, \\
a_{j} \frac{w_{j}=w_{A j}+w_{R j},}{d t}+\frac{3}{2} w^{2}+4 v_{l} \frac{w_{R j}}{a_{j}}=\frac{p_{g j}-p_{l}}{\rho_{l}^{0}}, w_{A j}=\frac{p_{g j}-p}{\rho_{l}^{0} C_{l} \alpha_{g j}^{1 / 3}}, \\
p_{l}=p_{0}+C_{l}^{2}\left(\rho_{l}^{0}-\rho_{l 0}^{0}\right), p_{g j}=\rho_{g j}^{0} B T_{g j}, \\
q_{g j}=\mathrm{Nu}_{g j} \lambda_{g j} \frac{T_{g j}-T_{0}}{2 a_{j}}, \frac{T_{g j}}{T_{0}}=\frac{p_{g j}}{p_{0}}\left(\frac{a_{j}}{a_{0 j}}\right)^{3}, \\
\kappa_{g j}=\frac{\lambda_{g j}}{C_{g j} \rho_{g j}} . \\
\mathrm{Pe}_{g j}=12\left(\gamma_{g j}-1\right) \frac{T_{0}}{\mid \mathrm{Pe}_{g j}}, \mathrm{Pe}_{g j} \geq 100 \\
10,
\end{gathered}
$$

Здесь все обозначения такие же, как в [15].

\section{Результаты расчетов}

В работах [18-20] отмечено, что характеристики детонационного процесса определяются только параметрами среды - характеристиками газовой фазы - дисперсностью пузырьков и их объемным содержанием, сортом газа внутри пузырьков, начальным давлением; характеристиками жидкости: вязкостью, скоростью звука и т. д. При этом структура волн пузырьковой детонации при изменении начального давлении остается качественно идентичной [18].

На рис. 1 представлены эпюры давления при распространении детонационной волны в пузырьковой жидкости, состоящей из активных (содержащих горючий газ) и пассивных (заполненных негорючим газом) пузырьков.

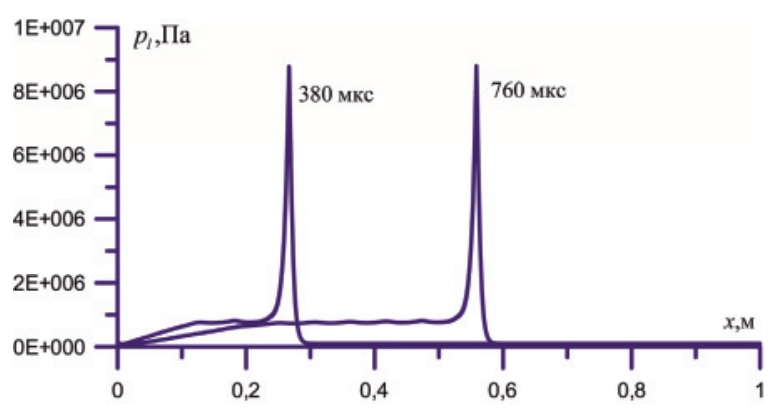

Рис. 1. Эпюры давления при распространении волны детонации в пузырьковой жидкости с следующими параметрами: $\rho_{10}^{\circ}=1130 \mathrm{Kr} / \mathrm{M}^{3}, C_{l}=1700 \mathrm{M} / \mathrm{C}_{,} v_{l}=6,0 \mathrm{MKM}^{2} / \mathrm{C}_{\text {, }}$ $\alpha_{g 01}=0,01, \quad \alpha_{g 02}=0,001, a_{g 01}=1,0 \mathrm{MM}, a_{002}=1,0 \mathrm{mM}$, $\rho_{g 01}^{0}=1,26 \mathrm{~K} \Gamma / \mathrm{M}^{3}, \rho_{g 02}^{0}=1,2 \mathrm{~K} \Gamma / \mathrm{M}^{3}, \lambda_{g 1}=2,49 \cdot 10^{-2} \mathrm{BT} /(\mathrm{M} \cdot \mathrm{K})$, $\lambda_{g 2}=1,63 \cdot 10^{-2} \mathrm{BT} /(\mathrm{M} \cdot \mathrm{K}), \gamma_{1}=1,35, \gamma_{2}=1,4, c_{91}=1,14 \mathrm{~K} Д$ Дж/(Kr.K), $c_{g 2}=1,0 \mathrm{KД} /(\mathrm{Kr} \cdot \mathrm{K}), T_{*}=1000 \mathrm{~K}, \Delta T=3200$, нижние индексы 1 и 2 соответствуют параметрам взрывчатого (ацетилено-кислородная смесь $\mathrm{C}_{2} \mathrm{H}_{2}+2,5 \mathrm{O}_{2}$ ) и инертного газа (воздух), жидкость - водоглицериновый раствор с объемной долей глицерина 0,5

Fig 1. Diagrams of pressure in propagation of a detonation wave in a bubble liquid with the following parameters: $\rho_{10}^{0}=1130 \mathrm{~kg} / \mathrm{m}^{3}, C_{1}=1700 \mathrm{~m} / \mathrm{s}, v=6,05 \mu \mathrm{m}^{2} / \mathrm{s}, \alpha_{g 01}=0,01$, $\alpha_{902}=0,001, a_{901}=1,0 \mathrm{~mm}, a_{902}=1,0 \mathrm{~mm}, \rho_{901}^{0}=1,26 \mathrm{~kg} / \mathrm{m}^{3}$, $\rho_{902}^{0}=1,2 \mathrm{~kg} / \mathrm{m}^{3}, \lambda_{91}=2,49 \cdot 10^{-2} \mathrm{~W} /(\mathrm{m} \cdot \mathrm{K}), \lambda_{\mathrm{g} 2}=2,63 \cdot 10^{-2} \mathrm{~W} /(\mathrm{m} \cdot \mathrm{K})$, $\gamma_{1}=1,35, \quad \gamma_{2}=1,4, \quad c_{g 1}=1,14 \mathrm{~kJ} /(\mathrm{kg} \cdot \mathrm{K}), \quad c_{g 2}=1,0 \mathrm{~kJ} /(\mathrm{kg} \cdot \mathrm{K})$, $T_{*}=1000 \mathrm{~K}, \Delta T=3200 \mathrm{~K}$, subscripts 1 and 2 correspond to the parameters of the explosive (acetylene-oxygen mixture $\mathrm{C}_{2} \mathrm{H}_{2}+2,5 \mathrm{O}_{2}$ ) and inert gas (air), liquid-glycerol solution with water solution with a volume of glycerol 0,5 parts

Из рис. 1 видно, что к моменту 380 мкс в пузырьковой жидкости, частично состоящей из инертных пузырьков, формируется детонационная волна амплитудой около 9,0 МПа. Это значение приблизительно на 1,0 МПа меньше, чем для случая распространения волн в однокомпонентной пузырьковой системе, состоящей только из пузырьков с активным газом с объемным содержанием $\alpha_{\mathrm{g} 0}=0,01$. К моменту 760 мкс детонационный солитон распространяется, не меняя амплитуды и протяженности. Как видно из рис. 1, скорость распространения детонационной волны составляет около 789 м/с. Кроме того, за детонационным солитоном видны отдельные пульсации давления, связанные с 
радиальной инерцией пузырьковой жидкости, изза наличия пузырьков с неактивным газом.

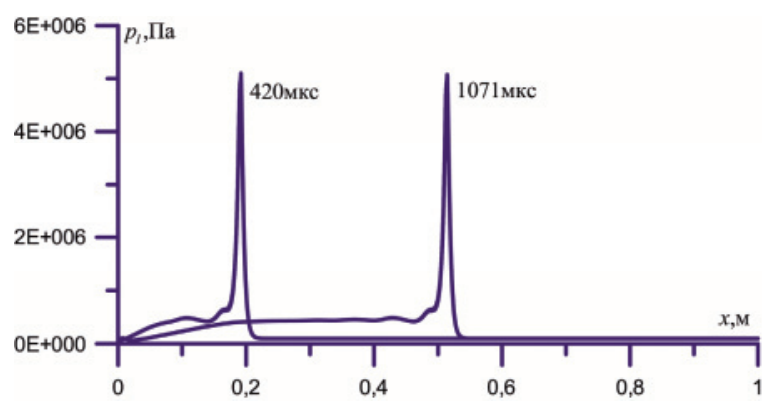

Рис. 2. Эпюры давления, $\alpha_{902}=0,01$, остальные параметры как на рис. 1

Fig. 2. Diagrams of pressure, $\alpha_{902}=0,01$, other parameters are the same as in Fig. 1

На рис. 2 представлено то же, что и на рис. 1 , но в этом случае объемное содержание пузырьков неактивного газа в 10 раз больше, чем для случая, представленного на рис. 1. Из рис. 2 видно, что амплитуда детонационного солитона в моменты 420 и 1071 мкс составляет около 5,0 МПа, что почти в два раза меньше амплитуды детонационной волны для случая, когда объемное содержание неактивного газа в пузырьковой среде составляет $\alpha_{\mathrm{g} 02}=0,001$. Скорость детонационной волны при этом составляет около $600 \mathrm{M} / \mathrm{c}$.

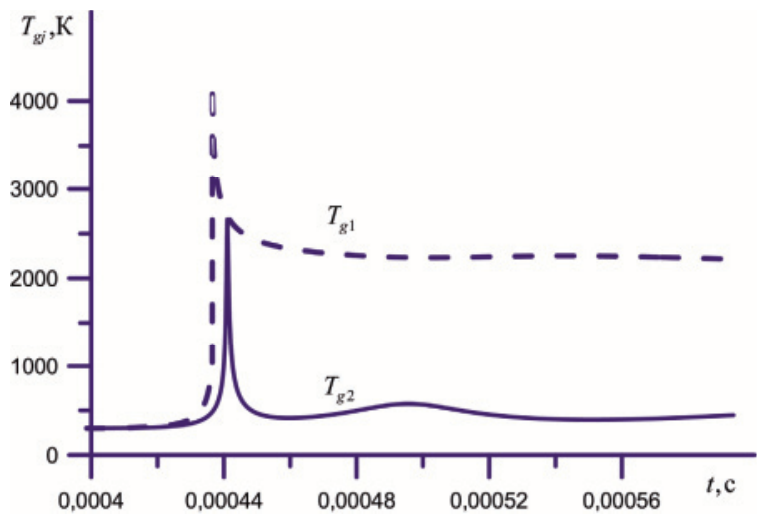

Рис. 3. Осциллограммы температуры газа в пассивных и активных пузырьках - сплошные и штриховые линии соответственно. Все параметры такие же, как на рис. 2

Fig. 3. Oscillograms of gas temperature in passive and active bubbles - solid and dashed lines, respectively. All parameters are the same as in Fig. 2

Расчетные осциллограммы для датчика D1, расположенного на расстоянии 0,2 м от границы $x=0$, представлены на рис. 3. Здесь штриховая линия соответствует температуре газа в активных (взрывчатых) пузырьках, сплошная - температуре газа в инертных. Из рис. 3 видно, что температура газа во взрывчатых пузырьках достигает значения 4200 К, в дальнейшем происходит уменьшение этого значения до 2500 К. Температура воздуха в инертных пузырьках в момент максимального сжатия увеличивается до значения более $2500 \mathrm{~K}$ и потом снижается до равновесного значения.

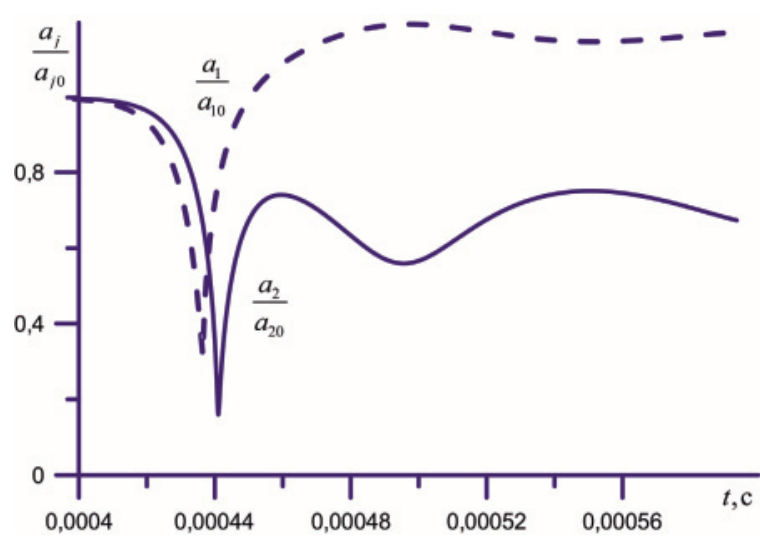

Рис. 4. Расчетные осциллограммы относительных радиусов активного (штриховые линии) и инертного (сплошные линии) пузырьков

Fig. 4. Calculated oscillograms of the relative radii of the active (dashed lines) and inert (solid lines) bubbles

На рис. 4 представлены расчетные осциллограммы относительных радиусов активного и пассивного пузырьков. Из рис. 4 видно, что при уменьшении радиуса активного пузырька почти в 3 раза происходит взрыв газа внутри пузырька, и он начинает расширяться. Из осциллограммы для инертного пузырька следует, что его радиус уменьшается почти в 10 раз.

На рис. 5 приведены зависимости скорости распространения волны детонации $D$ от концентрации химически неактивного газового компонента $\alpha_{\mathrm{g} 02}$ пузырьковой жидкости, при этом суммарное объемное содержание газовых содержаний $\alpha_{\mathrm{g} 0}=\alpha_{\mathrm{g} 01}+\alpha_{\mathrm{g} 02}$ является постоянной. Из рис. 5 видно, что расчетные значения скорости пузырьковой детонации достаточно близки к экспериментальным данным, т. е. математическая модель пузырьковой детонации, приведенная выше, достаточно хорошо описывает распространение детонационной волны в многокомпонентной пузырьковой жидкости по такому важному параметру пузырьковой детонации, как скорость детонационной волны.

Из рис. 5 видно, что общий вид зависимости $D\left(\alpha_{g 02}\right)$ при различных свойствах жидкой фазы качественно сходный - наличие в системе пузырьков неактивного газа приводит к снижению скорости распространения волны детонации. Снижение скорости обусловлено потерей энергии детонационной волны вследствие неравновесного теплообмена между газом и жидкостью и потерями, связанными с акустическим излучением.

Таким образом, наличие в системе пузырьков неактивного газа выступает фактором, обусловливающим дополнительные к имеющимся потери энергии волны пузырьковой детонации. Увеличение концентрации активного газового компонента (при данной неизменной общей концентрации газовой фазы) ведет к повышению скорости распространения волны детонации; а если возрастает концентрация пузырьков химически неактивного газа, то скорость детонационной волны падает. 


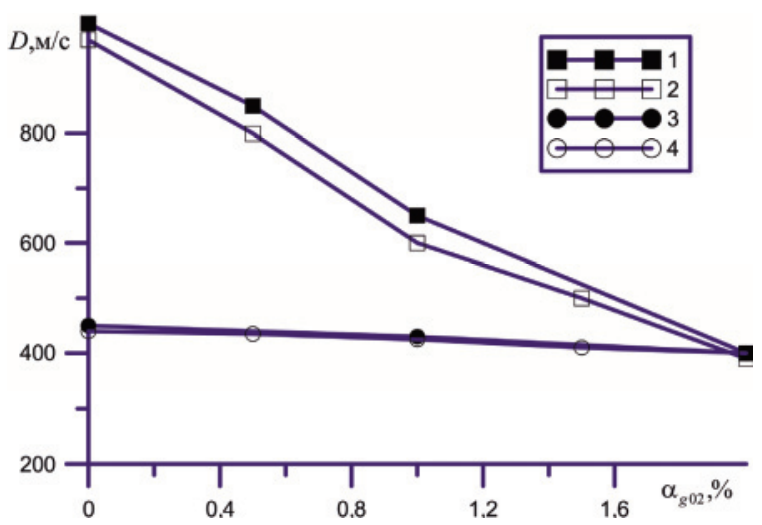

Рис. 5. Зависимость скорости волны пузырьковой детонации D в мноГокомпонентной пузырьКОвой жидкости от концентрации компонента неактивной газовой фазы $\alpha_{002}: \alpha_{00}=\alpha_{001}+\alpha_{02}=0,04, a_{901}=1,25 \mathrm{MM}, \quad a_{002}=1,25 \mathrm{MM}$, $\rho_{901}^{0}=1,26 \mathrm{Kr} / \mathrm{M}^{3}, \rho_{902}^{0}=1,78 \mathrm{Kr} / \mathrm{M}^{3}, \lambda_{91}=2,49 \cdot 10^{-2} \mathrm{BT} /(\mathrm{M} \cdot \mathrm{K})$, $\lambda_{g 2}=1,63 \cdot 10^{-2} \mathrm{BT} /(\mathrm{M} \cdot \mathrm{K}), \gamma_{1}=1,35, \gamma_{2}=1,67, c_{q 1}=1,14 \mathrm{KД} / \mathrm{K} /(\mathrm{Kr} \cdot \mathrm{K})$, $C_{g 2}=0,52 \mathrm{kД} /(\mathrm{Kг} \cdot K)$, линии 1 и 2 соответствуют экспериментальным данным и расчетным значениям, для следующих параметров системы: жидкость - во доглицериновый раствор с объемной долей глицери на 0,5, взрывчатый газ - ацетилено-кислородная смесь, неактивный газ - аргон (Ar), другие характеристики системы такие же, как на рис. 1. Линии 3 и 4 соответствуют экспериментальным данным и расчет ным значениям в случае, когда в качестве жидкой фа зы используется водоглицериновый раствор с объемной долей глицерина 0,25 со следующими параметрами: $\rho^{\circ}{ }_{10}=1065 \mathrm{Kr} / \mathrm{M}^{3}, C_{l}=1600 \mathrm{~m} / \mathrm{C}_{1} v_{1}=2,13 \mathrm{MKM}^{2} / \mathrm{C}$

Fig. 5. Dependence of the velocity of the bubble detonation wave $D$ in multicomponent bubble liquid on concentration of a component of the inactive gas phase $\alpha_{902}$. $\alpha_{g 0}=\alpha_{901}+\alpha_{g 02}=0,04, \quad a_{001}=1,25 \mathrm{~mm}, \quad a_{002}=1,25 \mathrm{~mm}$ $\rho_{901}^{0}=1,26 \mathrm{~kg} / \mathrm{m}^{3}, \rho_{902}^{0}=1,78 \mathrm{~kg} / \mathrm{m}^{3}, \lambda_{91}=2,49 \cdot 10^{-2} \mathrm{~W} /(\mathrm{m} \cdot \mathrm{K})$, $\lambda_{g 2}=1,63 \cdot 10^{-2} \mathrm{~W} /(\mathrm{m} \cdot \mathrm{K}), \gamma_{1}=1,35, \gamma_{2}=1,67, c_{g 1}=1,14 \mathrm{~kJ} /(\mathrm{kg} \cdot \mathrm{K})$, $c_{g 2}=0,52 \mathrm{~kJ} /(\mathrm{kg} \cdot \mathrm{K})$, lines 1 and 2 correspond to the experimental data and calculated values, liquid - water glycerine solution with a volume fraction of glycerin 0,5 , explosive gas - acetylene-oxygen mixture, inactive gas argon (Ar), the remaining parameters are the same as in Fig. 1. Lines 3 and 4 correspond to the experimental data and calculated values in the case when the liquid phase is a water glycerin solution with a volume fraction of glycerin 0,25 with the following parameters: $\rho_{10}^{0}=1065 \mathrm{~kg} / \mathrm{m}^{3}$, $C_{l}=1600 \mathrm{~m} / \mathrm{s}, v_{l}=2,13 \mu \mathrm{m}^{2} / \mathrm{s}$

Исследование динамики детонационных волн в пузырьковых жидкостях связано с анализом возможного взрыва в таких системах. Из экспериментов известно $[17,18]$, что первоначальное давление влияет на характеристики (скорость и амплитуду) детонационных волн в пузырьковой жидкости, например, увеличение первоначального давления приводит к увеличению диапазона амплитуд давления, которые способны инициировать детонацию. Поэтому необходимо также теоретическое исследование влияния первоначального давления в пузырьковой системе на характеристики волн пузырьковой детонации.

Зависимость давления детонационной волны от начального давления представлена на рис. 6 . Видно, что с увеличением первоначального давления на один порядок с 0,01 до 0,1 МПа происходит воз- растание пикового значения давления с 4,0 до 5,7 МПа. Отметим, что при понижении первоначального давлении от атмосферного (0,1 MПа) до 2 кПа давление в детонационной волне снижается до 3,0 МПа, что согласуется с экспериментальными данными [18]. Расчеты показали, что длительность детонационных волн при изменении начального давления пузырьковой среды практически не меняется.

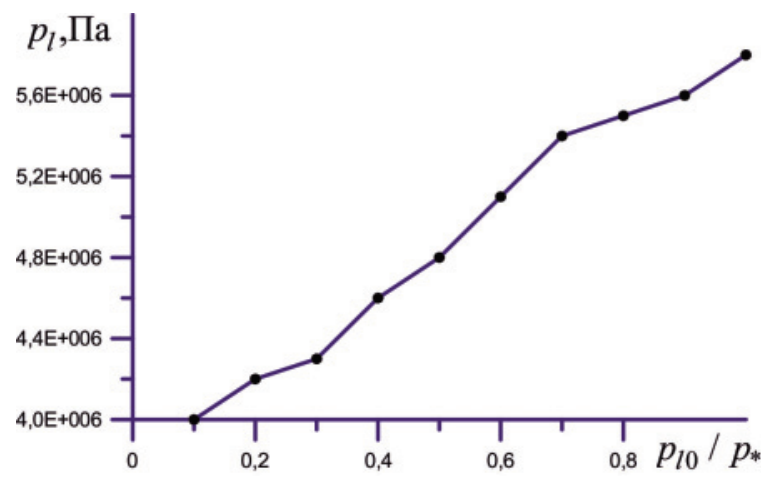

Рис. 6. Зависимости давления детонационных волн от начального давления $p_{10} / p_{*}, p_{*}=0,1$ МПа, параметры системы такие же, как для рис. 2

Fig. 6. Dependences of detonation waves pressure on the initial pressure $p_{10} / p_{*}, p_{*}=0,1 \mathrm{MPa}$, the system parameters are the same as for Fig. 2

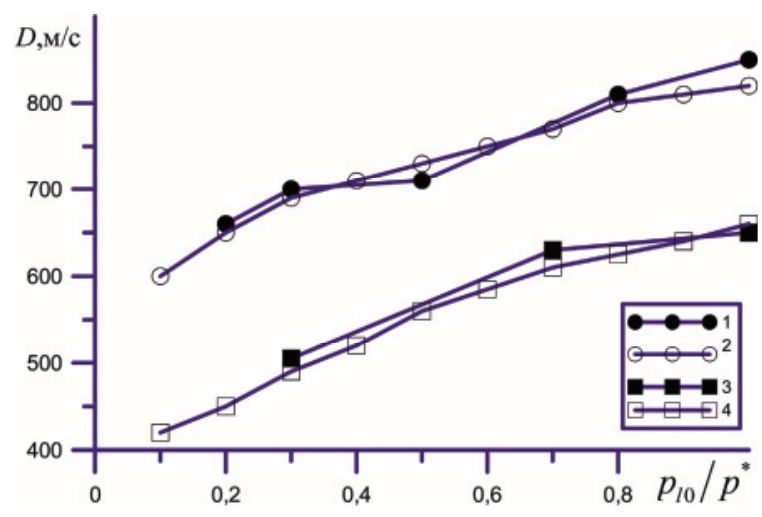

Рис. 7. Зависимости скорости детонационных волн D от начального давления $p_{10} / p_{*}$. Точки 1 и 2 соответствуют $\alpha_{g 0}=\alpha_{g 01}+\alpha_{g 02}=0,005+0,005=0,01,3$ и $4-\alpha_{g 0}=\alpha_{g 01}+\alpha_{g 02}=$ $=0,01+0,01=0,02, p_{*}=0,1$ МПа. Неактивный газ - не$\mathrm{OH}\left(\mathrm{N}_{2}\right)\left(\rho_{g 02}^{0}=1,25 \mathrm{K \Gamma} / \mathrm{M}^{3}, \lambda_{g 2}=2,4 \cdot 10^{-2} \mathrm{BT} /(\mathrm{M} \cdot \mathrm{K}), \gamma_{2}=1,4\right.$, $c_{g 2}=1,04 \mathrm{k}$ Дж/(кг.К)), другие характеристики системы такие же, как на рис. 5 (линии 1, 3 - эксперимент [18]; 2, 4 - расчет)

Fig. 7. Dependence of the velocity of detonation waves $D$ on the initial pressure ${ }_{10} / p_{*}$. Points 1 and 2 correspond to $\alpha_{00}=\alpha_{001}+\alpha_{002}=0,005+0,005=0,01,3$ and $4-\alpha_{00}=\alpha_{001}+\alpha_{002}=$ $=0,01+0,01=0,02, p_{*}=0,1 \mathrm{MPa}$. Inactive gas - neon $\left(\mathrm{N}_{2}\right)$ $\left(\rho_{g 02}^{0}=1,25 \mathrm{~kg} / \mathrm{m}^{3}, \quad \lambda_{\mathrm{g} 2}=2,4 \cdot 10^{-2} \mathrm{~W} /(\mathrm{m} \cdot \mathrm{K}), \quad \gamma_{2}=1,4\right.$, $\left.c_{g 2}=1,04 \mathrm{~kJ} /(\mathrm{kg} \cdot \mathrm{K})\right)$, other characteristics of the system are the same as in Fig. 5 (lines 1, 3 -experiment [18], 2, 4 - calculation)

На рис. 7 представлена зависимость скорости детонационных волн от начального давления. Видно, что с уменьшением начального давления многокомпонентных пузырьковых сред скорость рас- 
пространения детонационных волн снижается. Кроме того, можно утверждать, что зависимость скорости детонационной волны в многокомпонентной пузырьковой жидкости от начального давления близка к линейной.

\section{Заключение}

Зависимость параметров детонационных волн от начального давления многокомпонентных пузырьковых сред обусловлена уменьшением содержания энергии в таких средах. При уменьшении

\section{СПИСОК ЛИТЕРАТУРЫ}

1. Hasegawa T., Fujiwara T. Detonation in Oxyhydrogen Bubbled Liquids // Proc. $19^{\text {th }}$ Intern. Symp. on Combustion. - Hafia, 1982. - P. 675-683.

2. Sychev A.I., Pinaev A.V. Self-supported detonation in liquids with explosive gas bubbles // J. Appl. Mech. Techn. Physics. 1986. - № 1. - P. 133-138.

3. Pinaev A.V., Sychev A.I. Influence of physical-chemical properties of gas and a liquid on parameters and a condition of existence of a wave of a detonation in systems a liquid-gas bubbles // Physics of Combustion and Explosion. - 1987. - V. 23. - № 6. P. 76-84.

4. Сычев А.И. Трансформация детонационных волн на границе раздела пузырьковых сред // Журнал технической физики. 2011. - T. 81. - № 5. - C. 22-27.

5. Ljapidevskij V.J. About speed of a bubble detonations // Physics of Combustion and Explosion. - 1990. - V. 26. - № 4. P. $137-140$.

6. Gimaltdinov I.K., Arslanbekova R.R., Levina T.M. Features of the dynamics of postdetonation waves // Thermophysics and Aeromechanics. - 2016. - V. 23. - № 3. - P. 355-367.

7. Shagapov V.S., Vahitova N.K. Waves in a bubbly system at presence of chemical reactions in a gas phase // Physics of Combustion and Explosion. - 1989. - V. 25. - № 6. - P. 14-22.

8. Kedrinskij V.K. Hydrodynamics of explosion: experiment and models. - Novosibirsk: Siberian Branch of the Russian Academy of Science Publ., 2000. - 435 p.

9. Topolnikov A.S., Gimaltdinov I.K. Dynamics of detonation waves in a channel with variable cross section and filled with bubbly fluid // Thermophysics and Aeromechanics. - 2014. - V. 21. № 4. - P. 489-498.

10. Zhdan S.A. A detonation of a column of chemically active bubble medium // Physics of Combustion and Explosion. - 2003. V. 39. - № 4. - P. 107-112.

11. Dynamics of Detonation Waves in an Annular Layer of a Round Pipe / R. Bayazitova, I.K. Gimaltdinov, A.M. Kucher, V.Sh. Shagapov // Fluid Dynamics. - 2013. - V. 48. - № 2. - P. 201-210. начального давления многокомпонентной пузырьковой системы происходит снижение амплитуды и скорости детонационной волны. Таким образом, значение давления и концентрация пузырьков с инертным газом являются параметрами, с помощью которых можно управлять характеристиками (амплитудой и скоростью) детонационных волн.

Работа выполнена в рамках государственного задания Минобрнауки России в сфере научной деятельности, нолер для публикации 5.12863.2018/8.9. Работа также поддержана грантом РФФИ, номер проекта 17-41-020244.

12. 0 термоядерных процессах в кавитирующих пузырьках / Р.И. Нигматулин, Р.Т. Лэхи, Р.П. Талейархан, К.Д. Вест, Р.С. Блок // Успехи физических наук. - 2014. - Т. 184. № 9. - C. 947-960.

13. Theory of supercompression of vapor bubbles and nanoscale thermonuclear fusion / R.I. Nigmatulin, I.Sh., Akhatov A.S. Topolnikov, R.Kh. Bolotnova, N.K. Vakhitova, R.T. Lahey Jr., R.P. Taleyarkhan // Physics of Fluids. - 2005. - V. 17. - № 10. P. 107106(1)- 107106(31).

14. Nigmatulin R.I. Dynamics of Multiphase Media: V. 1-2. - New York, United States: Hemisphere Publ. Corp., 1990. - 507 p.

15. Gimaltdinov I.K., Kucher A.M. Detonation waves in a multicomponent bubble liquid // High Temperature. - 2014. - V. 52. № 3. - P. 411-416.

16. Сычев А.И. Детонационные волны в многокомпонентных пузырьковых средах // Физика горения и взрыва. - 1993. T. 29. - № 1. - С. 110-117.

17. Сычев А.И. Влияние начального давления пузырьковых сред на характеристики волн детонации // Журнал технической физики. - 2015. - Т.85. - № 4. - С. 126-129.

18. Сычев А.И. Влияние начального давления многокомпонентных пузырьковых сред на характеристики волн детонации // Журнал технической физики. - 2016. - Т. 86. - № 5. C. $15-19$.

19. Гималтдинов И. К., Столповский М. В., Левина Т. М. Влияние начального давления на характеристики детонационной волны в пузырьковой жидкости // Нефтегазовое дело: электрон. науч. журн. - 2017. - № 5. - C. 52-66. URL: http://ogbus.ru/issues/5_2017/ogbus_5_2017_p52-66_GimaltdinovIK_ru.pdf (дата обращения 01.11.2018).

20. Гималтдинов И.К., Левина Т.М. Особенности динамики детонационных волн в пузырьковой жидкости при прохождении границы "водоглицериновый раствор - масло» // Известия Томского политехнического университета. Инжиниринг георесурсов. - 2017. - T. 328. - № 8. - С. 55-63.

Поступила 23.11.2018 г.

\section{Информация об авторах}

Гималтдинов И.К., доктор физико-математических наук, профессор кафедры физики Уфимского государственного нефтяного технического университета, член-корреспондент Академии наук Республики Башкоргостан.

Левина T.M., кандидат технических наук, заведующая кафедрой общенаучных дисциплин Уфимского государственного нефтяного технического университета.

Kyчер A.M., преподаватель Башкирского государственного университета. 
UDC 532.329: 532.2.532

\title{
DEPENDENCE OF CHARACTERISTICS OF DETONATION WAVES IN MULTICOMPONENT BUBBLE LIQUID ON INITIAL PRESSURE
}

\author{
llias K. Gimaltdinov', \\ iljas_g@mail.ru \\ Tatyana M. Levina', \\ tattin76@mail.ru \\ Anastasia M. Kucher2, \\ kucheram85@mail.ru
1 Ufa State Petroleum Technological University, 1, Kosmonavtov street, Ufa, 450062, Russia.
2 Sterlitamak branch of Bashkir State University, \\ 49, Lenin avenue, Sterlitamak, 453100, Russia.
}

Relevance. Bubble liquids are a common working environment in a number of branches of the national economy. One of the most interesting processes is the propagation of detonation waves in a bubble liquid with a flammable gas inside the bubbles. Detonation waves can also propagate in a liquid with bubbles, partly consisting of inert gas. In this case, the presence of bubbles with inert gas is a certain control parameter for characteristics (amplitude, extent, propagation limits) of the detonation wave. In addition, there are experimental data on the influence of the initial pressure on the characteristics of bubble detonation waves in a multicomponent bubble liquid. This necessitates the investigation of detonation waves in multicomponent bubble systems for different values of the initial pressure.

The aim of the research is to study the dynamics of detonation waves in a bubble liquid, partially consisting of bubbles with inactive (non-flammable) gas at different initial pressures.

The object of research is detonation waves in a bubble liquid containing bubbles with an explosive (active) and non-flammable (inactive) gas.

The research method is based on the fundamental equations of the mechanics of multiphase media, which are solved numerically. The results of investigations on revealing the features of the propagation of detonation waves in a multicomponent bubble liquid at various initial pressures allowed us to draw conclusions and give recommendations. As the initial pressure of multicomponent bubble media decreases, the velocity of propagation of detonation waves decreases. The dependence of the detonation wave velocity in a multicomponent bubble liquid on the initial pressure is close to linear.

\section{Key words:}

Oil and gas production, explosion safety, detonation waves, bubble liquid, initial pressure.

The research was carried out within the State task of the Ministry of Education and Science of the Russian Federation in the field of research, release no. 5.12863.2018/8.9. The research was supported by the RFBR grant, project no. 17-41-020244.

\section{REFERENCES}

1. Hasegawa T., Fujiwara T. Detonation in 0xyhydrogen Bubbled Liquids. Proc. $19^{\text {th }}$ Intern. Symp. on Combustion. Hafia, 1982. pp. $675-683$.

2. Sychev A.I., Pinaev A.V. Self-supported detonation in liquids with explosive gas bubbles. J. Appl. Mech. Techn. Physics, 1986, no. 1. pp. 133-138.

3. Pinaev A.V., Sychev A.I. Influence of physical-chemical properties of gas and a liquid on parameters and a condition of existence of a wave of a detonation in systems a liquid-gas bubbles. Physics of Combustion and Explosion, 1987, vol. 23, no. 6, pp. 76-84.

4. Sychev A.I. Transformation of detonation waves at the interface between bubble media. Technical Physics, 2011, vol. 56, no. 5, pp. 611-615.

5. Ljapidevskij V.J. About speed of a bubble detonations. Physics of Combustion and Explosion, 1990, vol. 26, no. 4, pp. 137-140.

6. Gimaltdinov I.K., Arslanbekova R.R., and Levina T.M. Features of the dynamics of postdetonation waves. Thermophysics and Aeromechanics, 2016, vol. 23, no. 3, pp. 355-367.

7. Shagapov V.S., Vahitova N.K. Waves in a bubble system in the presence of gas-phase chemical reactions. Combustion, Explosion, and Shock Waves, 1989, vol. 25, no. 6, pp. 669-677.
8. Kedrinskij V.K. Hydrodynamics of explosion: experiment and models. Novosibirsk, Siberian Branch of the Russian Academy of Science Publ., 2000. 435 p.

9. Topolnikov A.S., Gimaltdinov I.K. Dynamics of detonation waves in a channel with variable cross section and filled with bubbly fluid. Thermophysics and Aeromechanics, 2014, vol. 21, no. 4, pp. 489-498.

10. Zhdan S.A. A detonation of a column of chemically active bubble medium. Physics of Combustion and Explosion, 2003, vol. 39, no. 4, pp. 107-112.

11. Bayazitova A.R., Gimaltdinov I.K., Kucher A.M., Shagapov V.Sh. Dynamics of Detonation Waves in an Annular Layer of a Round Pipe. Fluid Dynamics, 2013, vol. 48, no. 2, pp. 201-210.

12. Nigmatulin R.I., Lahey R.T., Block R.C., Taleyarkhan R.P., West C.D. On thermonuclear processes in cavitation bubbles. Physics-Uspekhi, 2014, vol. 57, no. 9, pp. 877-890.

13. Nigmatulin R.I., Akhatov I.Sh., Topolnikov A.S., Bolotnova R.Kh., Vakhitova N.K., Lahey R.T. Jr., Taleyarkhan R.P. Theory of supercompression of vapor bubbles and nanoscale thermonuclear fusion. Physics of Fluids, 2005, vol. 17, no. 10, P. 107106(1)-107106(31).

14. Nigmatulin R.I. Dynamics of Multiphase Media: vol. 1-2. New York, United States, Hemisphere Publ. Corp., 1990. 507 p. 
15. Gimaltdinov I.K., Kucher A.M. Detonation waves in a multicomponent bubble liquid. High Temperature, 2014, vol. 52, no. 3, pp. 411-416.

16. Sychev A.I. Detonation waves in multicomponent bubble media. Combustion, Explosion, and Shock Waves, 1993, vol. 29, no. 1, pp. 104-111.

17. Sychev A.I. Influence of the initial pressure in bubble media on the detonation wave parameters. Technical Physics, 2015, vol. 60 , no. 4, pp. 603-606.

18. Sychev A.I. Effect of the initial pressure of multicomponent bubble media on the characteristics of detonation waves. Technical Physics, 2016, vol. 61, no. 5, pp. 654-658.
19. Gimaltdinov I. K., Stolpovskiy M. V., Levina T. M. The influence of initial pressure on the characteristics of detonation waves in bubble liquids. Neftegazovoye delo, 2017, no. 5, pp. 52-66. In Rus. Available at: http://ogbus.ru/issues/5 2017/ogbus_5_2017_p52-66_GimaltdinovIK_ru.pdf (accessed 1 November 2018 ).

20. Gimaltdinov I.K., Levina T.M. Features of detonation wave dynamics in bubbly liquid on the border «hydroglyceric solution - oil». Bulletin of the Tomsk Polytechnic University. Geo Assets Engineering, 2017, vol. 328, no. 8, pp. 55-63.

Received: 23 November 2018.

\section{Information about the authors}

Ilias K. Gimaltdinov, Dr. Sc., professor, Ufa State Petroleum Technological University, corresponding member of the Academy of Sciences of the Republic of Bashkortostan.

Tatyana M. Levina, Cand. Sc., head of the department, Ufa State Petroleum Technological University.

Anastasia M. Kucher, college teacher Sterlitamak branch of Bashkir State University. 